Disease activity and damage accrual during the early disease course in a multinational inception cohort of patients with systemic lupus erythematosus

J. Nossent, E. Kiss, B. Rozman, G. Pokorny, P. Vlachoyiannopoulos, M. Olesinska, A. Marchesoni, M. Mosca, S. Påi, K. Manger, M. Schneider, H. Nielsen, R. van Vollenhoven and T. Swaak

Lupus 2010 19: 949 originally published online 7 April 2010

DOI: $10.1177 / 0961203310366572$

The online version of this article can be found at:

http://lup.sagepub.com/content/19/8/949

\author{
Published by: \\ (3)SAGE \\ http://www.sagepublications.com
}

Additional services and information for Lupus can be found at:

Email Alerts: http://lup.sagepub.com/cgi/alerts

Subscriptions: http://lup.sagepub.com/subscriptions

Reprints: http://www.sagepub.com/journalsReprints.nav

Permissions: http://www.sagepub.com/journalsPermissions.nav

Citations: http://lup.sagepub.com/content/19/8/949.refs.html

>> Version of Record - Jun 25, 2010

OnlineFirst Version of Record - Apr 7, 2010

What is This? 


\title{
Disease activity and damage accrual during the early disease course in a multinational inception cohort of patients with systemic lupus erythematosus
}

\author{
J Nossent $^{1}$, E Kiss ${ }^{2}$, B Rozman ${ }^{3}$, G Pokorny ${ }^{4}$, P Vlachoyiannopoulos ${ }^{5}$, M Olesinska ${ }^{6}$, A Marchesoni ${ }^{7}$, M Mosca ${ }^{8}$, \\ S Påi ${ }^{9}, K_{\text {Manger }}{ }^{10}$, M Schneider ${ }^{11}, \mathrm{H}_{\text {Nielsen }}{ }^{12}, \mathrm{R}$ van Vollenhoven ${ }^{13}$ and T Swaak ${ }^{14}$ \\ ${ }^{1}$ Department of Rheumatology, Institute of Clinical Medicine, University of Tromsø, Tromsø, Norway; ${ }^{2}$ Department of Internal Medicine, \\ Medical University of Debrecen, Debrecen, Hungary; ${ }^{3}$ Department of Rheumatology, Dr Peter Drzaj Hospital, Ljubljana, Slovenia; \\ ${ }^{4}$ First Department of Internal Medicine, Szent-Gyorgyi Medical University Centre, Szeged, Hungary; ${ }^{5}$ Department of Pathophysiology, \\ School of Medicine, National University of Athens, Athens, Greece; ${ }^{6}$ Department of Connective Tissue Diseases, Institute of Rheumatology, \\ Warsaw, Poland; ${ }^{7}$ Rheumatology Unit, Istituto Ortopedico Gaetano Pini, Milano, Italy; ${ }^{8}$ Universita degli Studi di Pisa, \\ Dipartimenti di Medicina Interna, Italy; ${ }^{9}$ Department of Internal Medicine, Faculty of Medicine, University of Tartu, Estonia; \\ ${ }^{10}$ Department of Internal Medicine III and Institute for Clinical Immunology, University of Erlangen-Nurnberg, Erlangen, Germany; \\ ${ }^{11}$ Medical Clinic, Department of Rheumatology, Heinrich-Heine University, Dusseldorf, Germany; ${ }^{12}$ Division of Rheumatology, \\ Herlev Hospital, University of Copenhagen, Denmark; ${ }^{13}$ Department of Rheumatology, Karolinska Institute, Stockholm, Sweden; \\ and ${ }^{14}$ Department of Rheumatology, Ikazia Ziekenhuis, Rotterdam, The Netherlands
}

\begin{abstract}
An inception cohort of patients with systemic lupus erythematosus from 14 European centres was followed for up to 5 years in order to describe the current early disease course. At inclusion patients ( $n=200,89 \%$ female, mean age 35 years, 97\% Caucasian, mean SLEDAI 12.2) fulfilled a mean of 6.5 ACR classification criteria. The most prevalent criteria were antinuclear $\mathrm{Ab}$ presence $(97 \%)$ followed by anti-dsDNA Ab (74\%), arthritis (69\%), leukocytopenia $(54 \%)$ and malar rash (53\%), antiphospholipid $\mathrm{Ab}(48 \%)$ and anti-synovial membrane $\mathrm{Ab}$ $(21.6 \%)$. Clinical signs of lupus nephritis (LN) were present in 39\% with biopsy-confirmed LN seen in $25 \%$. Frequent additional findings were hypocomplementaemia (54\%), anti-SSA Ab $(49 \%)$, alopecia (26\%) and Raynaud's phenomenon (31\%). There were few regional differences in disease presentation and management. One and 5-year survival rates were $99 \%$ and $97 \%$ respectively. During the mean follow-up of 4.1 years $25 \%$ entered a state of early disease quiescence by global physician assessment, but the overall risk of subsequent flare was $60 \%$. Maximum SLEDAI scores decreased over time, but $45 \%$ of patients accrued damage (SDI $\geq 1$ ) for which baseline presence of proteinuria and persistent disease activity were independent predictors. The results indicate minor differences in SLE presentation and treatment within various regions of Europe and a high diagnostic reliance on anti-dsDNA Ab. Despite early reductions in disease activity and improved mortality, the risk for disease flare and damage development is, however, still substantial, especially in patients not entering an early remission. Lupus (2010) 19, 949-956.
\end{abstract}

Key words: damage accrual; disease activity; Europe; inception cohort; systemic lupus erythematosus; therapy

\section{Introduction}

Despite the improvements in survival over the last decades, patients with systemic lupus erythematosus (SLE) remain at an increased risk for premature

Correspondence to: JC Nossent, Department of Rheumatology, University Hospital North Norway, PO Box 14, N-9038 Tromsø, Norway. Email: hans.nossent $@$ unn.no

Received 4 November 2009; accepted 22 February 2010 death. ${ }^{1-3}$ This risk is due to a complex of disease features, whose interaction is not well understood. Disease activity is a major determinant of prognosis as it can induce structural and functional organ damage directly through inflammation and thromboembolic events or indirectly through therapeutic measures associated with infectious and metabolic complications. ${ }^{4-8}$ The overall disease activity course remains unpredictable with flares continuing to occur well into the second decade of disease. ${ }^{9}$ Given the fact that SLE phenotypes are susceptible 
to genetic and/or geographic influences, ${ }^{10}$ that changes in SLE phenotype can occur within a single region ${ }^{11,12}$ and that patient management options are steadily expanding, ${ }^{13}$ there is a continued need for surveillance of the clinical characteristics and complications in SLE. This study describes the presentation and early disease course in a contemporary multinational European inception cohort and investigates current presentation and disease severity with regard to possible regional differences and the risk of organ damage and early mortality.

\section{Methods}

\section{Study design}

Using an existing infrastructure for SLE-related research, $8,9,1414$ rheumatology centres agreed in 1999 to assemble data on disease presentation in new onset SLE patients seen over a period of 5 years and provide follow-up data. After a core data set was defined, case record forms were developed and distributed. Data collection was performed by a designated physician at each centre with the use of predefined forms for baseline and follow-up data to summarize patient visits made in the previous calendar year. These forms were submitted to a study coordinator for case ascertainment and anonymous electronic data storage. This design is basically similar to the methods used in other multicentre cohort studies. ${ }^{15}$ This research received no specific grant from any funding agency in the public, commercial or not-forprofit sector. All patients were included in locally approved disease registries, and, while the frequency of follow-up visits was at the discretion of the attending physician, $>90 \%$ of patients were seen at least twice yearly with the observation period ending in 2005.

\section{Patients' data}

Only patients who fulfilled the revised ACR 1982 or the updated 1997 criteria $^{16,17}$ were included in the present analysis. For each patient the participating site provided baseline data (i.e. at time of fulfilment of ACR criteria) on demographics and disease manifestations as well as annual updates on scores for disease activity (SLEDAI) and accumulated damage SLICC/ACR Damage Index (SDI) ${ }^{18,19}$ and a global physician assessment (GPA) of disease activity. ${ }^{20}$ Disease duration was calculated from the time at least four ACR criteria were fulfilled. An arbitrary regional assignment of centres used in earlier studies $^{21}$ designated Germany $(n=12)$, The Netherlands $(n=10)$, Denmark $(n=2)$, Sweden $(n=4)$ and Norway $(n=11)$ as western, Italy $(n=29)$ and Greece $(n=18)$ as southern, Hungary $(n=54)$ as central and Poland $(n=18)$, Slovenia $(n=29)$ and Estonia $(n=13)$ as eastern. Results for laboratory and serological assays were obtained during routine workup at the participating centres, and abnormal results reflect data outside the local reference values.

\section{Statistics}

All figures represent median values (range) unless otherwise stated. Given the skewed distribution of most data, nonparametric test methods were used in data analysis, which used software program SPSS v 15.0. Numeric data were analysed by Mann-Whitney U test, while dichotomized data were expressed as odds ratios (OR) with $95 \%$ confidence intervals (CI). Predictors that were significantly associated with the relevant outcome $(\mathrm{SDI} \geq 1)$ were then entered into backward Cox regression models ( $p$ to enter $<0.1, p$ to stay $<0.05)$ to determine their independency. Resulting $p$ values $<0.05$ were considered statistically significant.

\section{Results}

\section{Descriptors}

Data for 220 patients were submitted, but 20 patients $(9 \%)$ were excluded as they did not fulfil four classification criteria upon case ascertainment. Baseline characteristics for the remaining patients $(n=200)$ showed no significant regional differences with regard to age, gender, pre-diagnostic symptomatic period or number of ACR criteria (Table 1). During the mean follow-up period of 4 years, four patients $(2.0 \%)$ died and 13 patients $(6.5 \%)$ were lost to follow-up.

\section{Baseline disease features}

Arthritis (69\%), leukocytopenia (54\%) and malar rash $(53 \%)$ were the predominant clinical ACR manifestations (Table 1). While 88 (39\%) patients had clinical signs of renal disease at baseline (i.e. proteinuria and/or cellular casts as defined by ACR definitions), LN was histologically confirmed in 52 $(25 \%)$. The reported WHO class distribution for biopsies was class II: 3\%, class III: $29 \%$, Class IV: $48 \%$ and class V: $10 \%$. Renal biopsies were 
Table 1 Patient characteristics at baseline. Figures indicate means with standard deviation unless otherwise indicated

\begin{tabular}{ll}
\hline Male/Female (\%) & $23 / 177(11 / 89)$ \\
Age at diagnosis (years) & $34.8 \pm 13$ \\
Western - central - southern - eastern & $20-33-24-23 \%$ \\
$\quad$ region* & \\
Time from onset to ACR classification & $28 \pm 23$ \\
$\quad$ (months) & \\
Number of ACR criteria fulfilled at scientific & $6.5 \pm 2.1$ \\
$\quad$ diagnosis & \\
Type of ACR criteria fulfilled at scientific & $6.5 \pm 2.1$ \\
diagnosis & \\
Malar rash & $53 \%$ \\
Photosensitivity & $51 \%$ \\
Discoid rash & $12 \%$ \\
Oropharyngeal ulcers & $15 \%$ \\
Arthritis & $69 \%$ \\
Pleuritis & $25 \%$ \\
Pericarditis & $17 \%$ \\
Proteinuria $>0.5 \mathrm{~g} /$ day & $39 \%$ \\
Haematuria & $36 \%$ \\
Sterile pyuria & $22 \%$ \\
Cellular casts & $23 \%$ \\
Seizures & $4 \%$ \\
Psychosis & $3 \%$ \\
Haemolytic anaemia & $13 \%$ \\
Leukocytopenia & $54 \%$ \\
Lymphocytopenia & $45 \%$ \\
Thrombocytopenia & $21 \%$ \\
Anti-dsDNA Ab & $78 \%$ \\
Anti-Sm Ab & $54 \%$ \\
Positive ANA & $22 \%$ \\
SLEDAI & $97 \%$ \\
\hline
\end{tabular}

*See methods section for geographical division. ANA, antinuclear Ab; anti-Sm, anti-synovial membrane; SLEDAI, Systemic Lupus Erythematosus Disease Activity Index.

not performed in the remaining 26 patients for reasons that included bleeding diathesis and refusal. Antinuclear Ab (ANA) was positive in 97\%; three of six patients with negative ANA had low avidity anti-dsDNA Ab, one had anti-SSA Ab and two patients had no other autoantibodies than antiphospholipid Ab (aPL). Anti-dsDNA Ab was present in $74 \%$ of patients; they were detected by Crithidia L. immunofluorescence (performed at 11 centres) in $76 / 168$ patients $(45 \%)$ tested, by Farr assay (three centres) in $36 / 62$ patients $(58 \%)$ tested and by enzyme-linked immunosorbent assay (seven centres) in $67 / 105$ patients $(64 \%)$ tested. The overall prevalence of other autoantibodies was $49 \%$ for anti-SSA, 48\% for aPL by any assay (aCL-G $43 \%$, aCL-M $16 \%$ and lupus anticoagulant $12 \%$ ) and anti-Sm Ab (21.6\%). Hypocomplementaemia (low C3 and/or C4 levels) was present in 54\% of patients; two-thirds of these patients were also positive for anti-dsDNA Ab. Sixteen patients $(8 \%)$
Table 2 Baseline prevalence of other disease manifestations than those included in current ACR classification criteria. Figures represent percentage of patients $(n=200)$

\begin{tabular}{llll}
\hline Feature & \multicolumn{3}{l}{ Feature } \\
\hline Alopecia & 25.5 & Arterial thrombosis & 2 \\
Purpura & 13.5 & Venous thrombosis & 6.5 \\
Teleangiectasias & 5 & Arterial hypertension & 13.5 \\
Urticaria & 6 & Raynaud's phenomena & 30.5 \\
Periungeal erythema & 7.5 & Avascular bone necrosis & 0.5 \\
Periorbital eryhema & 1.5 & Two spontaneous abortions & 2 \\
Livedo reticularis & 15.5 & Myocarditis & 4 \\
Retinopathy & 2 & Sterile endocarditis & 1.5 \\
Keratoconjunctivitis & 13.5 & Arrhythmia & 1 \\
Pneumonitis & 6 & Sterile ascites & 0.5 \\
Atelectasis & 1 & Intestinal vasculitis & 0.5 \\
Pulmonary fibrosis & 1 & Hepatosplenomegaly & 12 \\
Pulmonary hypertension & 1 & Lymphadenopathy & 18.5 \\
Arthralgia & 60.5 & Nonhaemolytic anaemia & 35.5 \\
Deforming arthritis & 1,5 & Aseptic meningitis & 1 \\
Tenosynovitis & 3 & Peripheral neuropathy & 6.5 \\
Myositis & 2.5 & Cranial neuropathy & 1 \\
Fibromyalgia & 9.5 & Cerebellar ataxia & 1 \\
Muscle weakness & 9 & TIA & 3.5 \\
Serum creatinine doubling & 3 & Transverse myelitis & 0.5 \\
GFR reduction $>50 \%$ & 6.5 & Organic brain syndrome & 4 \\
Biopsy-proven LN & 26.1 & Depression & 3 \\
& & Anti-RNP Ab & 19.8 \\
& & Anti-SSA Ab & 49.2 \\
& & Anti-SSB Ab & 26.8 \\
\hline & & & \\
& & & \\
& & &
\end{tabular}

GFR, glomerular filtration rate; TIA, transient ischaemic attack.

were aPL positive, while negative for anti-dsDNA or anti-Sm Ab.

While there was equal prevalence in the various regions of the majority of ACR classification criteria at baseline, there were significant differences in the frequency distribution of discoid rash, psychosis, leuko- and lymphocytopenia and autoantibody against Sm, PL and ds-DNA; however, no consistent pattern was discernible. A wide spectrum of other disease manifestations was present at baseline; non-haemolytic anaemia (35\%), Raynaud's phenomenon $(31 \%)$ and alopecia $(26 \%)$ were the most frequent objective findings (Table 2).

\section{Initial treatment}

Over the first 12 months of disease most patients $(83 \%)$ were treated with glucocorticosteroids, initiated as i.v. pulse methylprednisolone (MP) treatment in $33 \%$ of cases (Table 3). Concomitant treatment was with cytotoxic drugs $(54 \%)$, antimalarials $(51 \%)$ and non-steroidal antiinflammatory drugs (NSAIDs) $(28 \%)$. LN patients were more likely to receive pulse MP (OR 7.3, CI 3.6-14.4), cyclophosphamide (OR 20, CI 6.7-52) and azathioprine (OR 2.6, CI 1.3-5.2) and were 
Disease activity and damage accrual during the early disease course

J Nossent et al.

Table 3 Initial drug treatment in 200 patients with new onset SLE during the first year of disease

\begin{tabular}{ll}
\hline Oral corticosteroids & $83 \%$ \\
Cumulative steroid dose (mg/year) & 2635 \\
i.v. pulse corticosteroids & $33 \%$ \\
Antimalarials & $46 \%$ \\
Azathioprine & $25 \%$ \\
Cyclophosphamide (p.o. + i.v.) & $24.5 \%$ \\
Other immunosuppressant & $10.5 \%$ \\
NSAID & $28 \%$ \\
Aspirin & $4 \%$ \\
Warfarin & $1 \%$ \\
\hline
\end{tabular}

NSAID, non-steroidal anti-inflammatory drug.

less likely to receive antimalarials (OR 0.5 , CI 0.27 0.9 ) or NSAIDs (OR 0.2, CI 0.1-0.4).

There were no regional differences in the cumulative first year steroid dose or frequency of initial treatment with i.v. pulse MP, or azathioprine or NSAID use. However, use of pulse cyclophosphamide (central $12 \%$, west $16 \%$, south $27 \%$, east $33 \%, p=0.04)$ and antimalarial drugs $(24 \%$ in east, $64 \%$ in west and south, $p=0.002$ ) varied significantly. A total of four patients $(2 \%)$ were treated with anti-CD20.

\section{Disease activity course}

The baseline SLEDAI score was $12.2( \pm 9.8)$ and was lower in southern region patients (8.5 versus 12.9 for other regions, $p=0.012$ ), and median SLE scores decreased significantly over time ( $p<0.001$ for trend). Within the first year of disease 55 patients $(27.5 \%)$ achieved disease quiescence according to GPA while 145 patients $(62.5 \%)$ had persistent $(45 \%)$ or relapsing $(27.5 \%)$ disease activity during the first year of disease (Figure 2). There was no difference between patients with such early disease quiescence or persistently active disease in age at diagnosis (32 versus 29.5, $p=0.8$ ), number of ACR criteria (5.5 versus 6.0, $p=0.07$ ) or maximum steroid dose (39 versus 12 , $p<0.09$ ), but the cumulative steroid dose the first year (1860 versus $2750 \mathrm{mg}, p<0.06)$, initial SLEDAI (6.1 versus $12.5, p<0.001)$ and SDI at the end of the year ( 0 versus $1, p<0.001)$ were significantly lower in patients obtaining early quiescence. Approximately half of the patients (27/55; $49 \%$ ) achieving early quiescence maintained a state of remission (Figure 2A) throughout the entire follow-up period. In contrast, only $25 \%$ of patients with persistent disease activity in the first year subsequently reached a state of quiescence, $32 \%$ continued on a remitting-relapsing disease course and $43 \%$ never reached any state of remission
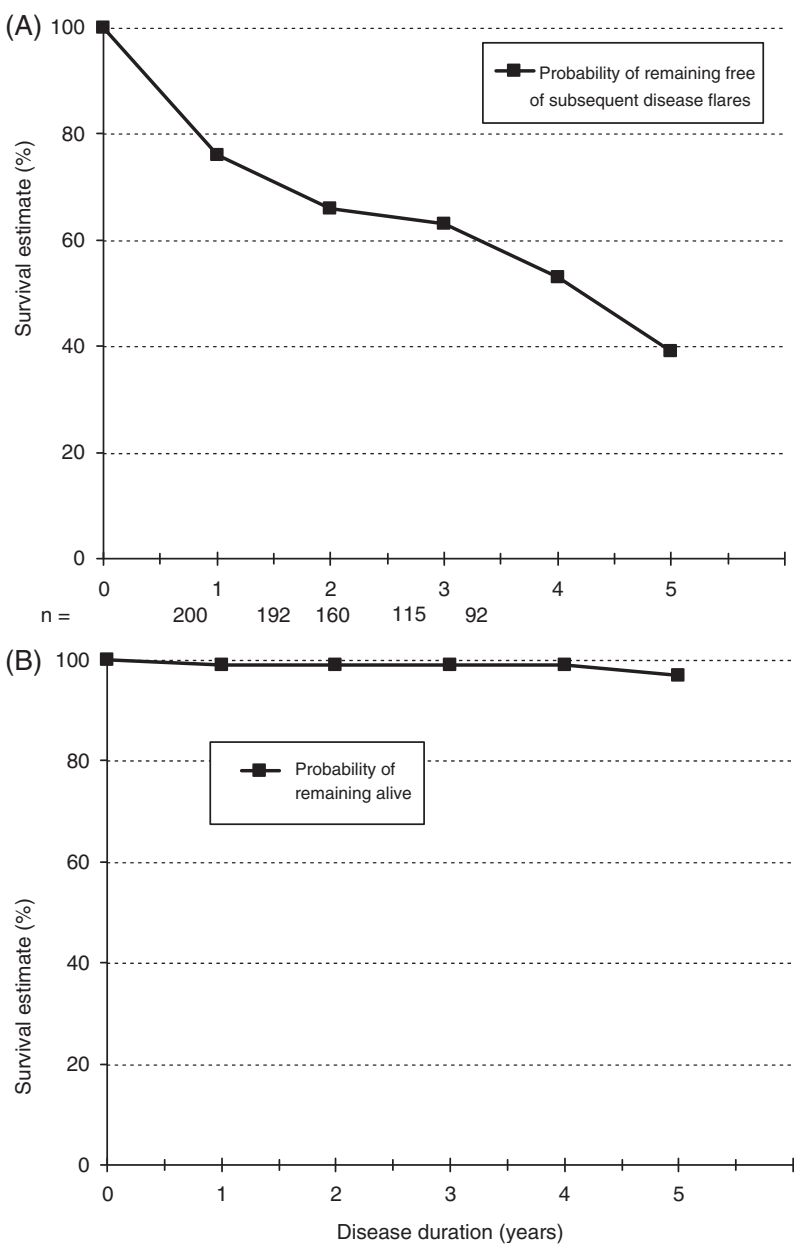

Figure 1 Kaplan-Meier curve showing the estimated probability of remaining free of disease flare, with non-surviving patients and patients with shorter follow-up censored from further observations (A) and the probability of survival during the first 5 years of disease in patients with new onset systemic lupus erythematosus with patients with shorter follow-up censored from further observations (B). Numbers (n) indicate the number of patients entering the given time period (i.e. still in study).

( $p<0.01$ for trend). Patients with early remission spent significantly less of their follow-up time with active disease (19.4 versus $77.9 \%$, $p<0.001)$, had lower annual relapse rates $(0$ versus $0.2, p<0.001)$, lower cumulative steroid dosage (1503 versus $3612 \mathrm{mg}, p<0.001)$, lower average SLEDAI ( 1 versus $8, p<0.001)$ and lower cumulative SDI scores ( 0 versus $1, p<0.001)$ than patients with persistent activity in the first year. Disease flares (defined as disease manifestations that necessitated changes in drug therapy other than NSAIDs) occurred in 91 patients $(46 \%)$ in total. The cumulative probability of having a disease flare was $24 \%$ in the first year and reached $61 \%$ by the fifth year of disease (Figure 1A). During 793 patient observation years a total of 

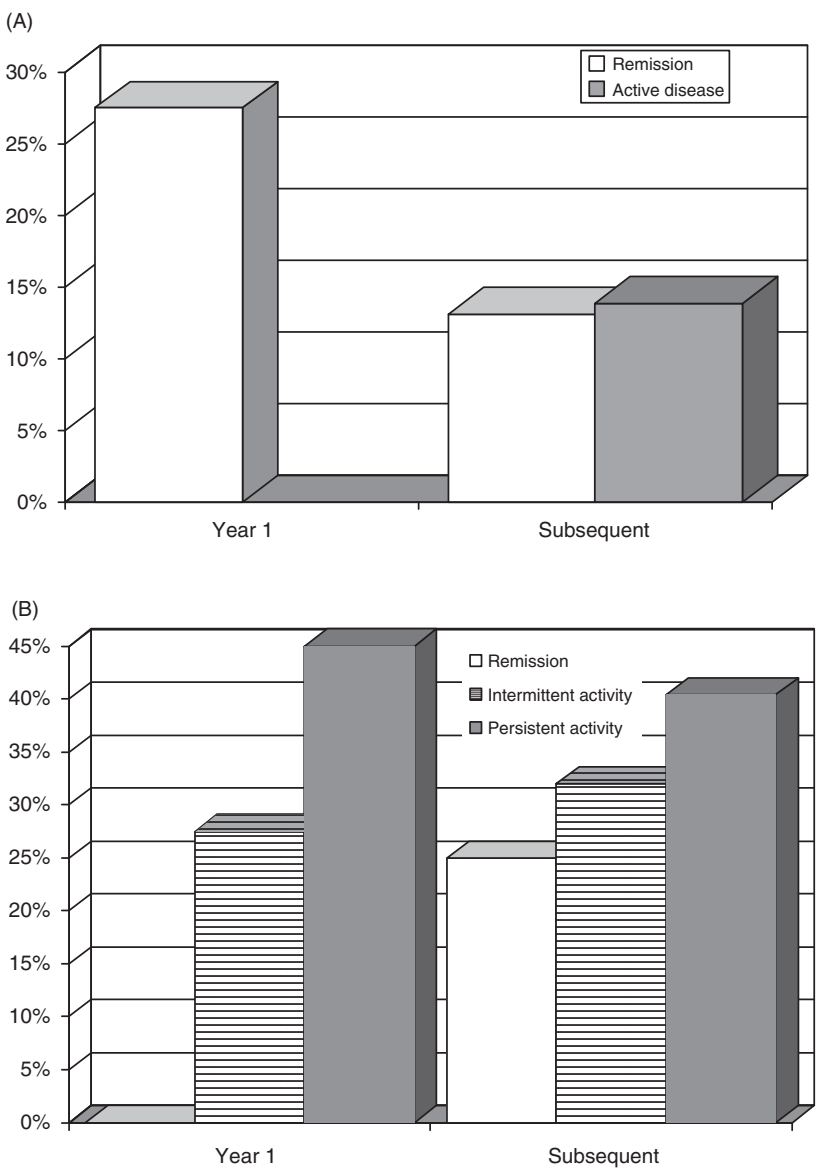

Figure 2 global physician assessment (GPA) of disease activity during the disease course for patients achieving disease quiescence in the first year (A) and for patients with persisting disease activity in the first year (B).

182 flares were recorded for an overall annual flare rate of 23/100 patient years. There was no significant association between flare prevalence and use of steroids or antimalarials, but cytotoxic drug use was associated with more flares $(p<0.05)$. Patients on antimalarial treatment spent more follow-up time in a quiescent disease state $(64 \%$ versus $48 \%, p<0.01)$.

\section{Organ damage development}

Damage (SDI score $\geq 1$ ) developed in $42 \%$ of patients during the observation period with the highest damage accrual occurring in the first 2 years of disease. Damage was moderate $(\mathrm{SDI}<3$ ) in $29 \%$ and severe $(\mathrm{SDI} \geq 3$ ) in $14 \%$. The highest SDI score of 18 was observed in a patient with fatal multi-organ involvement. SDI scores did not increase significantly after year one (1.2 after year one to 1.5 at last observation, $p>0.3$ ). One patient $(0.5 \%)$ developed a malignant (breast) tumour early on. End stage renal disease necessitating renal replacement therapy developed in three patients $(1.5 \%)$, while three additional patients had doubling of serum creatinine levels. Muscular atrophy was observed in 19 patients $(9.5 \%)$, deforming arthritis in 11 patients $(5 \%)$ and avascular bone necrosis in seven patients $(3.5 \%)$. Chronic cardiac conditions consisted of valvular disease in 16 patients $(8 \%)$, reduced left ventricle contractility $(7 \%)$ and coronary syndromes in $2 \%$ of cases. Other frequent types of vascular damage were venous thrombosis $(3.5 \%)$ and cerebral vascular accidents $(7.5 \%)$, which also were the main type of central nervous system (CNS) damage. Skin damage consisted of scarring alopecia in 15 patients $(7.5 \%)$ and chronic ulcers in 12 patients $(6 \%)$. Overall, the organ systems with the highest frequency of damage were musculoskeletal, renal, cardiovascular and neuropsychiatric systems. Significant predictors for SDI $>1$ by univariate analysis were baseline and subsequent maximum SLEDAI scores, presence of proteinuria, CNS involvement, vasculitis, anti-dsDNA Ab, hypocomplementaemia and treatment with i.v. pulse steroids and cyclophosphamide. Upon multivariate analysis only persistent disease activity and baseline renal affection remained independent predictors of damage accrual (Table 4).

\section{Mortality}

The direct causes of death in the four non-survivors were infectious complications (in two patients; one was on dialysis) and vascular events (one haemorrhagic brain infarct and one aortic dissection). The cumulative survival after 1 and 5 years was 99 and $97 \%$ respectively (Figure 1B). Given the low numbers of non-survivors a detailed analysis of predictors for survival was not feasible.

\section{Discussion}

This contemporary SLE inception cohort showed few regional differences in demographics and presentation of disease. Patient characteristics ( $89 \%$ female, age 35 years, diagnostic delay 28 months) corresponded well with those in similarly designed (observational multicentre cohort) studies. ${ }^{8,9,14,22-24}$ Similarly, the clinical burden of disease at diagnosis (6.5 ACR criteria, median SLEDAI >10) was comparable to that seen in the Caucasian LUMINA patient cohort $(n=71)$ (six ACR criteria and Systemic Lupus Activity Measure (SLAM) 8.5). 
Table 4 Multivariate Cox regression analysis model incorporating the baseline risk factors for damage development in SLE that were significant in univariate analyses. The hazard ratios indicate the increase in likelihood that the outcome occurs for cases in the relevant category compared with cases in the opposite category. Bold figures indicate predictors with independent prognostic value in the multivariate model

\begin{tabular}{lll}
\hline & \multicolumn{2}{l}{ Any damage $(S D I \geq 1)$} \\
\cline { 2 - 3 } & Hazard ratio & $95 \%$ CI \\
\hline Baseline SLEDAI & 1.0 & $0.9-1.1$ \\
Average annual SLEDAI & $\mathbf{1 . 2}$ & $\mathbf{1 . 1}-\mathbf{1 . 4}$ \\
Proteinuria & $\mathbf{1 . 3}$ & $\mathbf{1 - 1 . 6}$ \\
CNS (ACR definition) & 1.8 & $0.6-5.8$ \\
Vasculitis & 0.6 & $0.3-1.1$ \\
Anti-dsDNA Ab & 1.1 & $0.9-1.4$ \\
Hypocomplementaemia & 1.1 & $0.8-1.4$ \\
MP i.v. courses & 0.8 & $0.6-1.3$ \\
Cyclophosphamide use & 1.2 & $0.8-1.8$ \\
\hline
\end{tabular}

CNS, central nervous system; MP, methylprednisolone.

While the prevalence of clinical LN was $39 \%$, biopsy-verified $\mathrm{LN}$ was present in $25 \%$ of patients. These figures lie between the prevalence seen in Caucasians in LUMINA (32\%) and in Eurolupus $(22 \%)$. The biopsy findings were skewed towards more severe (i.e. proliferative) disease, and, while different reasons existed for not obtaining biopsies in the remainder of patients, a less aggressive diagnostic approach for presumed mesangial LN may be appropriate. ${ }^{25}$ Anti-dsDNA Ab were present at baseline in the large majority of patients $(74 \%)$ and constituted the most frequent SLE-specific classification criterion; although centres used different anti-dsDNA assays, this indicates an increasing reliance on anti-dsDNA Ab in the management of patients with suspected SLE. A similar high prevalence of anti-dsDNA $\mathrm{Ab}$ was also seen in the Eurolupus study (78\%) and far exceeds the $21 \%$ prevalence in the Caucasian patients in LUMINA. ${ }^{26}$ Whether this reflects difference in testing strategies or type of anti-DNA assays or a true difference in disease characteristics remains to be determined. The second most important serological feature was hypocomplementaemia in $54 \%$ of patients, which was accompanied by anti-dsDNA $\mathrm{Ab}$ in two-thirds of cases. Low complement levels are much more prevalent in SLE than in other connective tissue and inflammatory joint diseases, and, when combined with the presence of anti-dsDNA $\mathrm{Ab}$, will probably be highly specific for SLE as well, and this combination warrants consideration for inclusion in future classification criteria. ${ }^{27-29}$

SLE patients can present with a variety of manifestations that do not contribute significantly to the statistical process of disease classification. For patients and clinicians, however, these non-ACRcriteria manifestations are important in terms of disease burden and management. The spectrum of disease encountered at diagnosis (Table 2) is very much wider than the ACR criteria set. While arthralgia, alopecia, Raynaud's phenomena and anaemia of chronic disease were the most prevalent problems, thrombotic events were one of the severe, but less prevalent, manifestations. Antiphospholipid Ab have become part of the classification process for SLE, ${ }^{13}$ and the overall prevalence of aPL $(48 \%)$ agrees with other series. ${ }^{30,31}$ While inclusion of aPL as a classification criterion has not led to large changes in the clinical spectrum of SLE at diagnosis, ${ }^{12}$ they are likely connected to the remarkably high $(7.5 \%)$ overall frequency of ischaemic events in this cohort with a mean age of 35 years. The frequencies for vascular events in the first years of disease (cerebrovascular events $4 \%$, venous thrombosis $2 \%$ and angina pectoris $1 \%$ ) confirm the need for better markers to identify patients at vascular risk, as current aPL assays are not specific enough. ${ }^{32-34}$ The few classification criteria that showed a divergence in regional prevalence (discoid rash, autoantibodies other than anti-dsDNA) are not easily explained. While they may reflect methodological differences, they can also indicate true genetic or environmental influences on disease phenotype. ${ }^{35}$

Even though early disease activity was high, the subsequent disease course was characterized by a low mortality rate and a 5-year survival rate that was marginally higher than in the Eurolupus study; ${ }^{36}$ this nonetheless suggests that combined diagnostic and therapeutic developments may still have a positive impact on the short-term prognosis in SLE. This improved prognosis occurred despite the fact that the cumulative overall risk for a disease flare in the first 5 years of disease was over $60 \%$. This is in accordance with findings that disease flares remain a clinical challenge even after 15-20 years of disease. ${ }^{9,14}$ The overall flare rate of 23 per 100 patient years is at the lower end of reported flare rates, ${ }^{37,38}$ suggesting that both flare frequency and severity may be declining. Not achieving an early disease remission was associated with higer relapse rates and higher subsequent SLEDAI scores, indicating that the best window of therapeutic opportunity is in the first year of disease. While antimalarial drugs are important in maintaining disease quiescence, ${ }^{23}$ such treatment was less frequently used in two of the regions; this was not explained by differences in age, gender, initial SLEDAI or number of ACR criteria fulfilled 
(data not shown). While antimalarial drug use was associated with more follow-up time spent in disease quiescence in the whole cohort, we found no association between flare rates and lower regional use of antimalarials.

Despite improvements, control over disease activity was imperfect, as the risk of disease flare reached $60 \%$ and higher mean disease activity was the main predictor for overall damage development. Almost half of all patients accrued some organ damage during these earliest years of disease. This figure is comparable to the reported $40 \%$ damage prevalence 5 years into the disease. ${ }^{6,39}$ When organ-specific damage was analysed, it was clear that the main early damage was restricted to the musculoskeletal, CNS, cardiovascular and renal domains of SDI. Although damage in various domains has different implications, these types of organ damage are not necessarily a consequence of drug toxicity, a fact that is supported by a stable prevalence throughout the observation period of other drug-related co-morbidity, such as infectious diseases (data not shown). As organ damage is a significant risk factor for poorer prognosis, damage prevention becomes highly important; the current data indicate that better control of early disease activity is the main factor for the prevention of early damage. ${ }^{6,40-42}$ This will likely require use of immunosuppressive therapy for some time to come, even though the potential benefits of such treatment are offset by increased infectious complications. ${ }^{8}$ Thus, in addition to increased efforts for early diagnosis at a less severe disease stage, ${ }^{12}$ there is a clear need for less toxic but efficient ways of reducing early disease activity, such as through B-cell depletion or inactivation.

These results have several limitations. As data were collected in academic rheumatology centres throughout Europe, a selection bias may be present. Although one would expect this to be skewed toward patients with more severe disease, these results are not necessarily applicable to other regional settings. Also, different approaches to diagnosis and management cannot be wholly excluded; however, all centres have a long-standing research involvement in similar collaborative efforts. $8,9,14$ The collection of annual summary data may have underestimated disease activity, as patients with minor flares, other complications and related changes in medical treatment may not all have been referred to the study centre. Finally, the limited follow-up naturally precludes longerterm predictions.

In summary, SLE patients in Europe currently present with six defined ACR criteria and a high prevalence of anti-dsDNA antibodies. There is also a wide range of other manifestations that are not reflected by the classification criteria set and are better captured by disease activity scores such as SLEDAI. Patients with LN are most often managed with corticosteroids and cytotoxic drug initiation, with antimalarials and NSAIDs used in non-LN patients. This strategy is associated with low 5 year mortality and a swift decrease in disease severity. Nonetheless, $60 \%$ of patients had subsequent disease flare and 40\% accrued damage, both of which were most prevalent in patients not achieving an early disease remission.

\section{References}

1 Urowitz MB, Bookman AA, Koehler BE, Gordon DA, Smythe HA, Ogryzlo MA. The bimodal mortality pattern of systemic lupus erythematosus. Am J Med 1976; 60: 221-225.

2 Gladman DD, Urowitz MB. Prognosis, mortality, and morbidity in systemic lupus erythematosus. In: Wallace DJ, Hahn BH (eds), Dubois' Lupus Erythematosus, 6th edn. Philadelphia, PA: Lippincott Williams \& Wilkins; 2002. p. 1255-1273.

3 Bernatsky S, Boivin JF, Joseph L, et al. Mortality in systemic lupus erythematosus. Arthritis Rheum 2006; 54: 2550-2557.

4 Bjornadal L, Lofstrom B, Yin L, Lundberg IE, Ekbom A. Increased cancer incidence in a Swedish cohort of patients with systemic lupus erythematosus. Scand J Rheumatol 2002; 31: 66-71.

5 Bjornadal L, Yin L, Granath F, Klareskog L, Ekbom A. Cardiovascular disease a hazard despite improved prognosis in patients with systemic lupus erythematosus: results from a Swedish population based study 1964-95. J Rheumatol 2004; 31: 713-719.

6 Becker-Merok A, Nossent HC. Damage accumulation in systemic lupus erythematosus and its relation to disease activity and mortality. $J$ Rheumatol 2006; 33: 1570-1577.

7 Nived O, Jonsen A, Bengtsson AA, Bengtsson C, Sturfelt G. High predictive value of the Systemic Lupus International Collaborating Clinics/American College of Rheumatology damage index for survival in systemic lupus erythematosus. J Rheumatol 2002; 29: 1398-1400.

8 Nossent J, Cikes N, Kiss E, et al. Current causes of death in systemic lupus erythematosus in Europe, 2000-2004: relation to disease activity and damage accrual. Lupus 2007; 16: 309-317.

9 Swaak AJ, van den Brink HG, Smeenk RJ, et al. Systemic lupus erythematosus. Disease outcome in patients with a disease duration of at least 10 years: second evaluation. Lupus 2001; 10: 51-58.

10 Alarcon GS, Roseman J, Bartolucci AA, et al. Systemic lupus erythematosus in three ethnic groups: II. Features predictive of disease activity early in its course. LUMINA Study Group. Lupus in minority populations, nature versus nurture. Arthritis Rheum 1998; 41: 1173-1180.

11 Stahl-Hallengren C, Jonsen A, Nived O, Sturfelt G. Incidence studies of systemic lupus erythematosus in Southern Sweden: increasing age, decreasing frequency of renal manifestations and good prognosis. J Rheumatol 2000; 27: 685-691.

12 Eilertsen GO, Becker-Merok A, Nossent JC. The influence of the 1997 updated classification criteria for systemic lupus erythematosus: epidemiology, disease presentation, and patient management. $J$ Rheumatol 2009; 36: 552-559.

13 Eisenberg R, Albert D. B-cell targeted therapies in rheumatoid arthritis and systemic lupus erythematosus. Nat Clin Pract Rheumatol 2006; 2: 20-27.

14 Swaak AJ, van den Brink HG, Smeenk RJ, et al. Systemic lupus erythematosus: clinical features in patients with a disease duration 
of over 10 years, first evaluation. Rheumatology (Oxford) 1999; 38: 953-958.

15 Cervera R, Khamashta MA. Epidemiology of systemic lupus erythematosus at the change of the millennium: lessons from the Euro-Lupus and the LUMINA projects. Lupus 2006; 15: 1-2.

16 Tan EM, Cohen AS, Fries JF, et al. The 1982 revised criteria for the classification of systemic lupus erythematosus. Arthritis Rheum 1982; 25: 1271-1277.

17 Hochberg MC. Updating the American College of Rheumatology revised criteria for the classification of systemic lupus erythematosus. Arthritis Rheum 1997; 40: 1725.

18 Gladman DD, Goldsmith CH, Urowitz MB, et al. The Systemic Lupus International Collaborating Clinics/American College of Rheumatology (SLICC/ACR) Damage Index for Systemic Lupus Erythematosus International Comparison. J Rheumatol 2000; 27: 373-376.

19 Bombardier C, Gladman DD, Urowitz MB, Caron D, Chang CH. Derivation of the SLEDAI. A disease activity index for lupus patients. The Committee on Prognosis Studies in SLE. Arthritis Rheum 1992; 35: 630-640.

20 Barr SG, Zonana-Nacach A, Magder LS, Petri M. Patterns of disease activity in systemic lupus erythematosus. Arthritis Rheum 1999; 42: 2682-2688.

21 Swaak AJ, van de BH, Smeenk RJ, Manger K, et al. Incomplete lupus erythematosus: results of a multicentre study under the supervision of the EULAR Standing Committee on International Clinical Studies Including Therapeutic Trials (ESCISIT). Rheumatology (Oxford) 2001; 40: 89-94.

22 Cervera R, Khamashta MA, Font J, et al. Morbidity and mortality in systemic lupus erythematosus during a 5-year period. A multicenter prospective study of 1,000 patients. European Working Party on Systemic Lupus Erythematosus. Medicine (Baltimore) 1999; 78: 167-175.

23 Alarcon GS. Lessons from LUMINA: a multiethnic US cohort. Lupus 2008; 17: 971-976.

24 Urowitz MB, Gladman D, Ibanez D, et al. Clinical manifestations and coronary artery disease risk factors at diagnosis of systemic lupus erythematosus: data from an international inception cohort. Lupus 2007; 16: 731-735.

25 Bertsias G, Ioannidis JP, Boletis J, Bombardieri S, et al. EULAR recommendations for the management of systemic lupus erythematosus. Report of a Task Force of the EULAR Standing Committee for International Clinical Studies Including Therapeutics. Ann Rheum Dis 2008; 67: 195-205.

26 Alarcon GS, Friedman AW, Straaton KV, et al. Systemic lupus erythematosus in three ethnic groups: III. A comparison of characteristics early in the natural history of the LUMINA cohort. LUpus in MInority populations: NAture vs. Nurture. Lupus 1999; 8: 197-209.

27 Heinlen LD, McClain MT, Merrill J, et al. Clinical criteria for systemic lupus erythematosus precede diagnosis, and associated autoantibodies are present before clinical symptoms. Arthritis Rheum 2007; 56: 2344-2351.

28 Merrill JT, Buyon JP. The role of biomarkers in the assessment of lupus. Best Pract Res Clin Rheumatol 2005; 19: 709-726.

29 Kasitanon N, Magder LS, Petri M. Predictors of survival in systemic lupus erythematosus. Medicine (Baltimore) 2006; 85: $147-156$.

30 McMahon MA, Keogan M, O'Connell P, Kearns G. The prevalence of antiphospholipid antibody syndrome among systemic lupus erythematosus patients. Ir Med J 2006; 100: 296-298.

31 Tarr T, Lakos G, Bhattoa HP, Szegedi G, Shoenfeld Y, Kiss E. Primary antiphospholipid syndrome as the forerunner of systemic lupus erythematosus. Lupus 2007; 16: 324-328.

32 McClain MT, Arbuckle MR, Heinlen LD, et al. The prevalence, onset, and clinical significance of antiphospholipid antibodies prior to diagnosis of systemic lupus erythematosus. Arthritis Rheum 2004; 50: 1226-1232.

33 Bertolaccini ML, Hughes GR, Khamashta MA. Revisiting antiphospholipid antibodies: from targeting phospholipids to phospholipid binding proteins. Clin Lab 2004; 50: 653-665.

34 Abu-Shakra M, Gladman DD, Urowitz MB, Farewell V. Anticardiolipin antibodies in systemic lupus erythematosus: clinical and laboratory correlations. Am J Med 1995; 99: 624-628.

35 Dahlgren J, Takhar H, Anderson-Mahoney $\mathrm{P}$, et al. Cluster of systemic lupus erythematosus (SLE) associated with an oil field waste site: a cross sectional study. Environ Health 2007; 6: 8.

36 Cervera R, Khamashta MA, Font J, et al. Morbidity and mortality in systemic lupus erythematosus during a 10-year period: a comparison of early and late manifestations in a cohort of 1,000 patients. Medicine (Baltimore) 2003; 82: 299-308.

37 van den Berg L, Nossent H, Rekvig O. Prior anti-dsDNA antibody status does not predict later disease manifestations in systemic lupus erythematosus. Clin Rheumatol 2006; 25: 347-352.

38 Tseng CE, Buyon JP, Kim M, et al. The effect of moderate-dose corticosteroids in preventing severe flares in patients with serologically active, but clinically stable, systemic lupus erythematosus: findings of a prospective, randomized, double-blind, placebocontrolled trial. Arthritis Rheum 2006; 54: 3623-3632.

39 Alarcon GS, Roseman JM, McGwin Jr G, et al. Systemic lupus erythematosus in three ethnic groups. XX. Damage as a predictor of further damage. Rheumatology (Oxford) 2004; 43: 202-205.

40 Nossent JC. SLICC/ACR Damage Index in Afro-Caribbean patients with systemic lupus erythematosus: changes in and relationship to disease activity, corticosteroid therapy, and prognosis. $J$ Rheumatol 1998; 25: 654-659.

41 Rahman P, Gladman DD, Urowitz MB, Hallett D, Tam LS. Early damage as measured by the SLICC/ACR damage index is a predictor of mortality in systemic lupus erythematosus. Lupus 2001; 10: 93-96

42 Gladman DD, Urowitz MB, Rahman P, Ibanez D, Tam LS. Accrual of organ damage over time in patients with systemic lupus erythematosus. J Rheumatol 2003; 30: 1955-1959. 